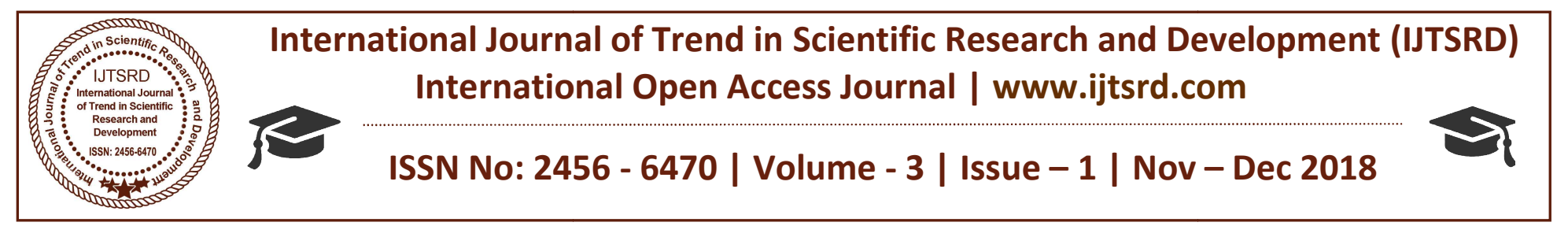

\title{
Intelligent Fall Detection Using Statistical Features and Machine Learning
}

\author{
Hephzibah Thomas ${ }^{1}$, Thyla B ${ }^{2}$ \\ ${ }^{1}$ PG Student, ${ }^{2}$ Assistant Professor \\ Department of Electronics and Communication, \\ KCG College of Technology, Chennai, Tamil Nadu, India
}

\begin{abstract}
Falls have become common nowadays among the elderly. It has been noted by the World Health Organization (WHO) that approximately one out of 3 elderly people aged above 65 living alone tend to fall and the rate can increase in the coming years. Many ideas have been proposed and worked out including using of inertial sensors, accelerometer and gyro meter. This paper proposes a method where the video from the camera is processed and the features are extracted. The features are extracted using HOG and statistical approach. The database contains fall and daily activities and Support Vector Machine (SVM) is used for classification which gives an accuracy of $100 \%$.
\end{abstract}

KEY WORDS: Image Processing, Machine Learning, HOG, Statistical feature, SVM

\section{INTRODUCTION}

Medical admission due to impact of fall is increasing innumerably. According to WHO people aged above 65 years tend to fall often. Apart from the elderly those with muscular dystrophy, osteoporosis, Parkinson disease, fits are prone to fall and extreme care needs to be given as a person laying unattended for a prolonged period of time after a fall may have physical as well as physiological ailments. Due to which research has been done on fall detection using many techniques including wearable sensors, kinect sensor, video surveillance etc. Wearable sensors are obtrusive for patients and the elderly people as they would not be able to wear it often due to their daily activities. Fall detection using video surveillance has been studied to a far extend and many systems have been implemented with a good accuracy. In [1], different statistical features have been studied. In [2],
GMM is used for background subtraction. Motion, Height-Width ratio, distance between top and mid centre of rectangle are used to detect a fall. In [3], an ellipse approximation and Motion History Image (MHI) method is used. In [4], Randomized Decision Tree (RDT) algorithm is proposed for the key joint extraction and SVM classifier is applied. In [5], deep learning followed by transfer learning is used. In [6], Histograms of Oriented Gradients (HOG), Local Binary Pattern (LBP) and feature extracted by the Deep Learning Framework Caffe are combined to form a new augmented feature and the feature was named HLC. In [7], five different classification algorithms were implemented and evaluated based on their accuracy, sensitivity, and specificity achieved. In [8], median filter is used for background subtraction and disparity map, moment functions, centroid etc are used to detect the fall. In [9], the detector operator utilized the fast region $\mathrm{CNN}$ trained parameters to consider the detected object's position. In [10], GMM and MHI are applied followed by calculation of acceleration and angular acceleration.

\section{PROPOSED METHOD}

The proposed method is used to detect a fall using a combination of HOG, statistical features and Machine learning concepts. 10 fall videos and 10 non fall videos are trained and Support Vector Machine (SVM) is used for classification. Gaussian Mixture Model (GMM) is used to extract the foreground features or subtract the background. HOG combined with 9 statistical features are used to extract features that contatin information to detect the fall. Once the fall is detected an alarm is generated and an email is send to the doctor along with the captured image. 
A. WORKFLOW

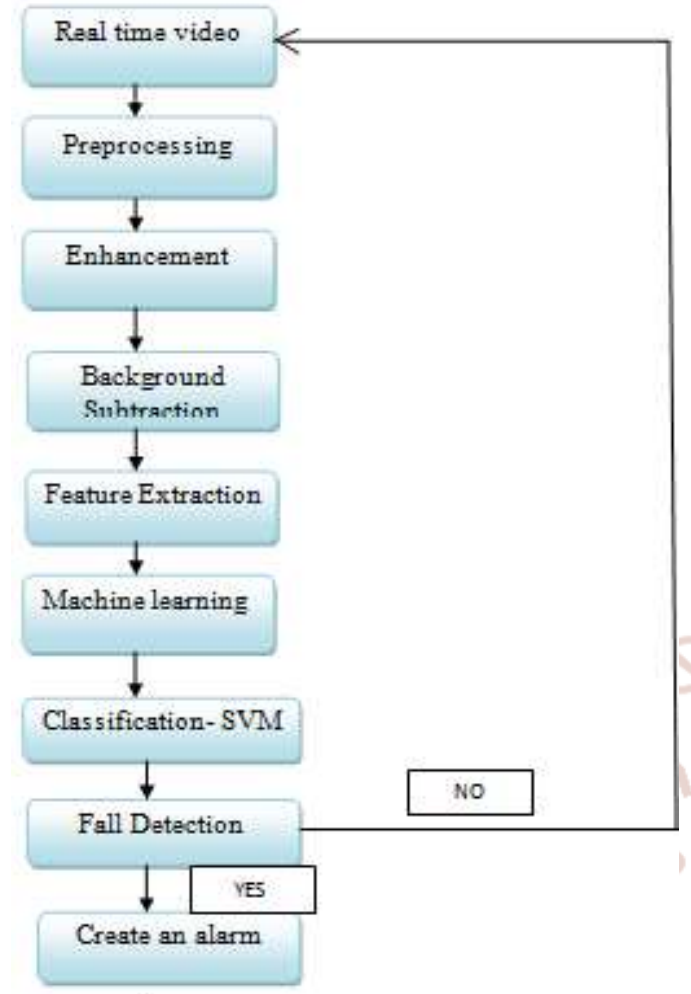

Fig 1: Workflow of Proposed System

\section{B. HARDWARE}

Processor Type : Pentium -IV

Speed $\quad: 2.4 \mathrm{GHZ}$

Ram

$: 4 \mathrm{~GB}$

Hard disk

: 20 GB HD

Camera

: IP CAMERA

\section{SOFTWARE:}

Operating System : Windows 8.1

Programming : Matlab2016b

\section{DATABASE:}

The dataset for training and testing was obtained from the available database: https://cvl.tuwien.ac.at/research/cvl-databases/falldatabase/.

\section{E. PRE-PROCESSING}

The video input from the IP Camera is pre-processed before it can be given to the neural network. The composite video is digitized in to a series of frames containing RGB images. Each RGB image is then converted to its gray scale image.

\section{F. ENHANCEMENT}

The images are resized which is a scale transformation. Here each pixel in the output image is mapped to its corresponding point maps in the input image. Once the resize is done interpolation is performed.

$\mathrm{J}\left(\mathrm{r}^{\prime}, \mathrm{c}^{\prime}\right)=\mathrm{I}(\mathrm{r}, \mathrm{c}) \cdot(1-\Lambda \mathrm{r}) \cdot(1-\Lambda \mathrm{c})+\mathrm{I}(\mathrm{r}+$

$1, c) \cdot \Lambda \mathrm{r} \cdot(1 \mathrm{c})+\mathrm{T}(\mathrm{r}, \mathrm{c}+1) \cdot(1-\Lambda \mathrm{r}) \cdot \Lambda \mathrm{c}+$

$\mathrm{I}(\mathrm{r}+1, \mathrm{c}+1) \cdot \Lambda \mathrm{r} \cdot \Lambda \mathrm{c}$

The frames are resized into $256 * 256$ sized images. The resized images are the filtered to remove noise. The noise removal is done by Gaussian filter. Applying a Gaussian filter is convolving the image with a Gaussian function.

$$
G(x, y)=\frac{1}{2 \pi \sigma^{2}} e^{-\frac{x^{2}+y^{2}}{2 \sigma^{2}}}
$$

The image is also segmented to get the useful information and avoid that is not. The gray images are converted to binary for further processing. This is done by applying a threshold value and all the pixels greater than the threshold are assigned the value 1 (white) and the other pixels are assigned 0 (black) The background image is subtracted using the Gaussian Mixture Models (GMM) method. The foreground image, being the moving object is extracted from the image. The foreground detector will require a number of frames to initialize GMM.

$$
w_{j}^{(i)}=\frac{g_{j}(x) \phi_{j}}{\sum_{l=1}^{k} g_{l}(x) \phi_{l}}
$$

This equation is applied to every cluster and example forming a matrix.

\section{G. FEATURE EXTRACTION}

A combination of Histogram of Oriented Gradients (HOG) and 9 statistical features is used to extract the required features.

Histogram of Oriented Gradients (HOG) is used to detect objects using edge detection. It has been found to be extremely useful in detecting human. HOG along with the statistical features. It is a feature descriptor that counts gradient orientation in localized portions. It is analysed by intensity gradients and edge directions

The first step in HOG is to divide the images into blocks and each block is divided into smaller regions called cells. For each pixel within the cell the horizontal and vertical gradients are obtained and this is done by using 1-D Sobel operators. 
HOG along with statistical features plays a better role in efficient detection of fall. 9 statistical features are extracted here. This includes mean, standard deviation, entropy, variance, smoothness, kurtosis, skewness.

\section{H. CLASSIFICATION}

Support Vector Machine (SVM) is used to classify the falls and ADL. SVM is a separation of classes. A hyper plane is used to separate the classes. Regularization, gamma and kernel are important in SVM.

The learning of the hyper plane is done by using linear algebra.

$\mathrm{f}(\mathrm{x})=\mathrm{B}(0)+\operatorname{sum}(\mathrm{ai} *(\mathrm{x}, \mathrm{xi}))$

Margin is a separation between the 2 classes and labels are given based on a threshold value.

In SVM we tend to increase the margin between the data points and the hyper plane

Gradient update for no misclassification is given by,

$$
w=w-\alpha \cdot(2 \lambda w)
$$

Gradient update for misclassification is,

$$
w=w-\alpha \cdot(2 \lambda w)
$$

Based on all the above a fall is detected.

\section{EXPERIMENT AND RESULT}

The proposed system is implemented using MATLAB and experimented on 10 videos including fall and ADL out of which 7 were fall videos and 3 were ADL videos.

Table 1: Accuracy for proposed method

\begin{tabular}{|c|c|c|c|}
\hline Activities & $\begin{array}{c}\text { Fall } \\
\text { Detected }\end{array}$ & $\begin{array}{c}\text { Fall not } \\
\text { detected }\end{array}$ & Accuracy \\
\hline FALL & 7 & 0 & $100 \%$ \\
\hline ADL & 0 & 3 & $0 \%$ \\
\hline
\end{tabular}

Table 2: Comparison of accuracy with existing method

\begin{tabular}{|c|c|c|}
\hline Method & True Positive & True Negative \\
\hline PROPOSED & $100 \%$ & $0 \%$ \\
\hline EXISITING & $95.2 \%$ & $3.33 \%$ \\
\hline
\end{tabular}

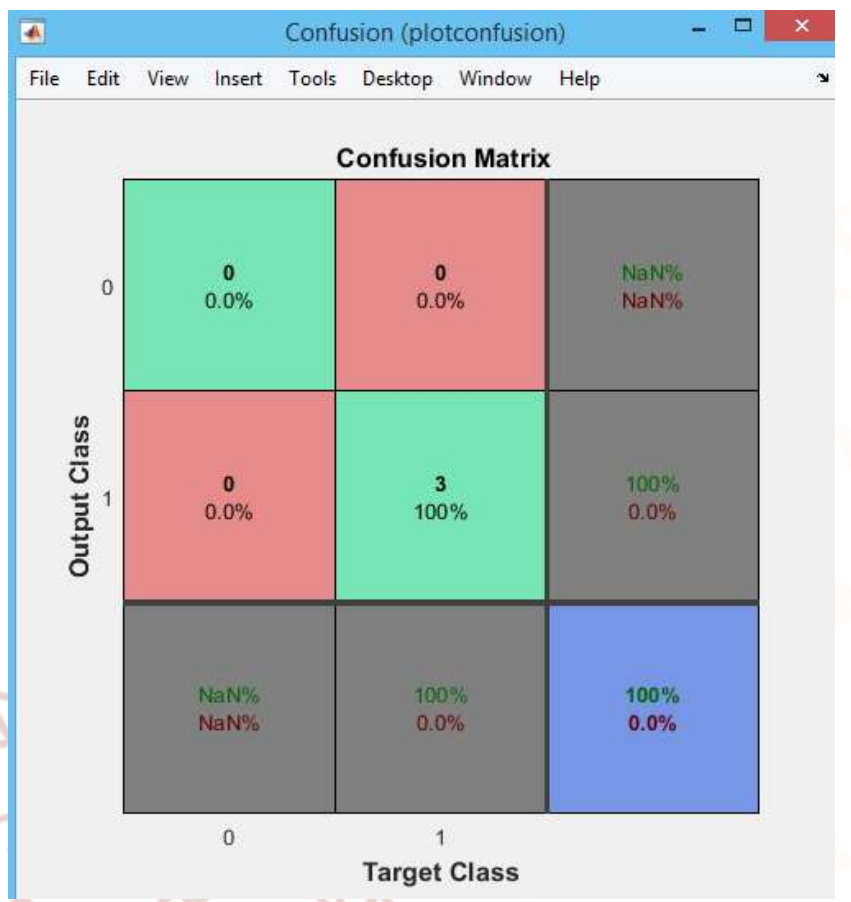

Fig 2: Confusion matrix showing $100 \%$ accuracy

\section{CONCLUSION}

The proposed paper uses Gaussian Mixed Model (GMM) for foreground extraction and Histogram Of Oriented Gradients (HOG) along with statistical features is used to extract features. Datasets are trained and Support Vector Machine (SVM) is used to classify and detect a fall. This was experimented on 10 videos and gave an accuracy of $100 \%$ which is better that the existing method. Future works will be implemented to increase the number of datasets and analyse the performance.

\section{REFERENCES}

1. Vijay Kumar1, Priyanka Gupta," Importance of Statistical Measures in Digital Image Processing", International Journal of Emerging Technology and Advanced Engineering, Volume 2, Issue 8, August 2012

2. Subhash Chand Agrawal; Rajesh Kumar Tripathi ; Anand Singh Jalal, "Human fall detection from an indoor video surveillance", 2017 8th International Conference on Computing, Communication and Networking Technologies (ICCCNT), Pages 1-5, 2017

3. G M Basavaraj; Ashok Kusagur, "Vision based surveillance system for detection of human fall", 2nd IEEE International Conference on Recent Trends in Electronics, Information \& Communication Technology (RTEICT), Pages 1516-1520, 2017 
4. Zhen-Peng Bian; Junhui Hou ; Lap-Pui Chau; Nadia Magnenat-Thalmann, "Fall Detection Based on Body Part Tracking Using a Depth Camera", IEEE Journal of Biomedical and Health Informatics, Volume 18, Issue 2, Pages $430-439,2015$

5. Lesya Anishchenko, "Machine learning in video surveillance for fall detection", Ural Symposium on Biomedical Engineering, Radio electronics and Information Technology (USBEREIT), Pages 99102,2018

6. Kun Wang; Guitao Cao; Dan Meng; Weiting Chen; Wenming Cao, "Automatic fall detection of human in video using combination of features", EEE International Conference on Bioinformatics and Biomedicine (BIBM), Pages 1228-1233, 2016

7. Pranesh Vallabh; Reza Malekian; Ning Ye; Dijana Capeska Bogatinoska, Fall detection using machine learning algorithms, "24th International Conference on Software, Telecommunications and Computer Networks (SoftCOM)", September 2016
8. LeiYang, Yanyun Ren, WenqiangZhang,"3D depth image analysis for indoor fall detection of elderly people", Digital Communications and Networks, Volume 2, Issue 1, 2016

9. Yi-Zeng Hsieh; Yu-Lin Jeng, "Development of Home Intelligent Fall Detection IoT System Based on Feedback Optical Flow Convolutional Neural Network", IEEE Access , Volume 6, Pages 60486057,2018

10. Chih-Yang Lin; Shang-Ming Wang; Jia-Wei Hong ; Li-Wei Kang ; Chung-Lin Huang, "Visionbased detection through shape features", IEEE Second International Conference on Multimedia Big Data (BigMM), Pages 237 - 240 , 2016

11. Zishan Zahidul Islam, Syed Mahir Tazwar, Md. Zahidul Islam, Seiichi Serikawa, and Md. Atiqur Rahman Ahad, "Automatic fall detection of unsupervise elderly people using smartphone", 5th IIAE International Conference on Intelligent Systems and Image Processing, 2017 